\title{
Combinatorial Approach of poly-D, L-lactide Encapsulated Quercetin, Piperlongumine and Curcumin Inhibits Tumour Growth in Balb/c Mice, the Colon Cancer Model
}

RAM DHAN YADAV ( $\nabla$ rdy.rrt@gmail.com )

Panjab University https://orcid.org/0000-0002-8625-0259

Abha Chaudhary

Government College

\section{Research Article}

Keywords: Quercetin, Piperlongumine, Curcumin, Banyan, PLA, Colon cancer

Posted Date: March 30th, 2021

DOI: https://doi.org/10.21203/rs.3.rs-346842/v1

License: (c) (1) This work is licensed under a Creative Commons Attribution 4.0 International License. Read Full License 


\section{Abstract}

Colon cancer has become an extreme danger to human lives due to high frequency and mortality around the world. The natural formulations based on the nanoparticles have been found promising in terms of cost, procedure and side effects. In this study, for the first time, three natural molecules- quercetin, piperlongumine and curcumin were encapsulated in biodegradable poly-D,L-lactide nanoparticles to check their anticancer potential against the colon cancer (DMH-DSS). The pure molecules, blank and loaded nanoparticles were given to the colon cancer model (BALB/c mice) and evaluated the anticancer potential of the nano-formulations. Ficus benghalensis (Banyan) coated nanoparticles maintained the detectable concentration of quercetin, piperlongumine and curcumin in colon even in liver, kidney and serum after $8 \mathrm{~h}$ of administration. In case of sugar coated NPs, quercetin, piperlongumine and curcumin were detected in colon, kidney and liver. Further, it was found that the coating of nanoparticles surface with leaf extract and dextrose sugar enhanced the potential of the formulation. This newly synthesized formulation can have the potential to be explored further as anticancer drug against the colon cancer.

\section{Introduction}

Colon cancer is the most commonly found malignancy around the world and is the 3rd leading reason of cancer related mortality in men and women. The causes of this type of cancer includes genetic and environment factors and unhealthy lifestyle, it is found more in those consuming red meat and saturated fats while found less in those taking vegetarian diet [1]. Generally the available colon cancer treatment involves conventional chemotherapy using 5 -fluorouracil, however, $40 \%$ of the cases found to be resistant to it [2]. The major cause for mortality are fast reappearance and metastasis regardless of best treatment. There is a dire need to create proficient natural therapeutic agents and to identify the phenomenon undergoing in colon cancer growth recurrence and metastasis to grow more effective prognostic biomarkers and remedial targets is earnestly required.

Naturally occurring molecules or phytochemicals are known as potential therapeutic agents and shown protection against cancer; the reason for thirst for researchers for continuously finding novel natural molecules. The natural molecules or precursors in drug findings have indicated possible helpful application in disease treatment. Quercetin is one of the significant flavonoids present in our everyday diet, which shows potential against malignant growth through displaying pro-apoptotic effect and restraining advancement of various human tumours [3]. Piperlongumine is naturally synthesized in long pepper and is reported to selectively kill tumour cells by elevation ROS levels in various cancer models including colon cancer. Curcumin is a well-known hydrophobic polyphenol and is characteristic in Chinese medications. In pharmacology, numerous studies have indicated that curcumin could be utilized as anti-oxidant, anti-inflammatory, and against malignant growth. But, the serious issue in its application is its very low bioavailability notwithstanding with its efficiency in cancer therapy. Quercetin, piperlongumine, and curcumin are well known natural molecules for their anticancer, antioxidant, and anti-inflammatory properties $[3,4]$. These molecules have shown significant anticancer activities, however, they have reduced bioavailability owing to low solubility in water and fast exodus from the body. 
According to recent literature there have been found several reports of effectiveness of single natural molecules against cancer [5]. The mechanism of action of the curcumin, quercetin and piperlongumine were quite different in different cell lines. Curcumin block the factor-alpha-induced nuclear translocation and DNA binding of NF-KB, supress the c-Fos subunit of AP-1. While the quercetin has been shown to block the tumour necrosis factor-alpha-induced nuclear translocation and DNA binding of NF-KB in human myelomonoblastic leukemia (ML-1a) cells. Curcumin and quercetin also alter the other key pathways which have direct or indirect roles in cancer control.

Lodi et al. used the combinatorial approach against the cancer cell lines and results were much better as compared to individual molecules [6]. Natural molecules support the anticancer profile of other molecules in compatibility way $[7,8]$. There have been major advances in the use of nanoparticles (NPs) as therapeutic platforms for the treatment of prostate, ovarian, breast and lung cancers [9]. The drug delivery systems using NPs aim to produce prolonged NPs circulation, easy delivery and efficient accumulation in the tumour cells. Nevertheless, despite the high morbidity and mortality associated with colon cancer, the clinical development of NPs for treatment of colon cancer remains limited.

Plant materials were used for the synthesis of green NPs to overcome the various issues associated with toxicity of NPs [10]. In present study we have used the aqueous leaf extract (LEs) of Banyan (Ficus benghalensis) in the synthesis of biogenic PLA (Poly-D,L-lactide) NPs and encapsulated three molecules curcumin, quercetin and piperlongumine using solvent evaporation method. Loaded NPs showed excellent loading of molecules, good stability and sustained release. Synthesized NPs (loaded and blank) were tested on colon cancer model (DMH-DSS) developed in BALB/c male mice. This experimental study used combinatorial approach of three natural molecules for the very first time with high loading on single type NPs for their anticancer profile on the developed colon cancer model. Furthermore efforts were done to make a coating of sugar on the surface of NPs at the time of synthesis to maximise the accumulation of molecules in colon cancer.

\section{Materials And Methods}

\section{Chemicals and materials}

Piperlongumine was purchased from Indofine Chemical Company (Hillsborough, NJ, USA). Curcumin and quercetin were purchased from the SRL (India). Leaves of the Ficus benghalensis were obtained from the Punjab university campus, Chandigarh (India). Dextran sulphate sodium salt (DSS) and DMH were purchased from the MP Biomedicals (Solon, OH, USA). Poly-D,L-lactide (PLA, mw=75000-120000) was purchased from the Sigma Aldrich and HPLC grade acetonitrile and water purchased from the SRL (India).

\section{Experimental animals}

Five to six week old male BALB/c weighing 20-25 g were procured from Central Animal House, Panjab University, Chandigarh, India. All experimental protocols were first approved by Institutional Ethics 
Committee (PU/IAEC/S/14/47) and conducted according to the guidelines of Indian National Science Academy for the use and care of experimental animals. The animals were housed in ventilated polypropylene cages and acclimatized for one week in the animal room before the commencement of the study. The animals were fed on standard mouse chow pellet diet supplied by Aashirwad industries, Punjab, India and water ad libitum.

\section{Preparation of leaf extracts}

For the formation of leaf extracts (LEs), leaf materials were crushed with the help of mortar and pestle into fine powder and $5.0 \mathrm{~g}$ material was dissolved in the $100 \mathrm{~mL}$ distilled water and heated at $60^{\circ} \mathrm{C}$. After cooling the remaining $50 \mathrm{~mL}$ material was centrifuged $\left(10000 \mathrm{rpm}, 20 \mathrm{~min}, 4^{\circ} \mathrm{C}\right)$ and filtered through a $0.22 \mu \mathrm{m}$ filter to get finally freshly prepared LEs for the synthesis PLA NPs. The extracts were stored at 4 ${ }^{\circ} \mathrm{C}$ for further use.

\section{Synthesis of LEs-mediated QPC-PLA NPs}

Poly-D,L-lactide (mw=75 000-120000), was used for the synthesis of polymeric NPs using solvent evaporation method. Fifty five milligram PLA was dissolved in $2 \mathrm{~mL}$ dichloromethane (DCM) with $6 \mathrm{mg}$ each of three molecules curcumin (C), quercetin (Q) and piperlongumine (P) and allowed to sonicate for $40 \mathrm{~s}$. After sonication $4 \mathrm{~mL}$ of LEs was added and again sonicated for $40 \mathrm{~s}$ to form emulsion and finally diluted to $80 \mathrm{~mL}$ using distilled water. DCM was evaporated using rota-vapour for $20 \mathrm{~min}$ and synthesized loaded NPs were centrifuged at $4^{\circ} \mathrm{C}$ for $15 \mathrm{~min}$. Similar conditions were followed for the synthesis of blank NPs. After separating the NPs using centrifuge, NPs were re-dissolved in the distilled water (4 mL). The freshly prepared NPs were twicely washed and ready for the characterization. The blank and molecules loaded LEs mediated NPs were named as LEs-PLA NPs and LEs-QPC-PLA NPs respectively.

\section{Coating of sugar and its evaluation on the surface of NPs}

For the encapsulation of sugar on the surface of NPs, the blank and loaded NPs were incubated overnight with the dextrose sugar solution $(2 \mathrm{mg} / \mathrm{mL})$. Anthrone test method was used for the estimation of sugar on the surface of LEs-S-PLA NPs and LEs-S-QPC-PLA NPs. The sugar coated LEs-S-PLA NPs and LEs-SQPC-PLA NPs were centrifuged and incubated with the Anthrone for sugar estimation and scanned at 620 $\mathrm{nm}$ using spectrophotometer. The sugar concentration was calculated on the surface of LEs-S-PLA NPs and LES-S-QPC-PLA NPs using formula (1).

Test concentration $=\frac{\mathrm{OD} \text { of Sample }}{\mathrm{OD} \text { of Blank }} \times$ Std. concentration $\times$ dilution factor

$\mathrm{OD}=$ optical density

\section{Morphological Characterization of blank and loaded NPs}


High resolution transmission electron microscopy (HRTEM, FEl, Netherlands) was used for shape and size measurement of LEs-PLA NPs and LEs-QPC-PLA NPs. Negative stain phosphotungstic acid was used to coat the surface of NPs and allow to dry at room temperature on copper grid. The images were obtained with a Tecnai, Twin 200 kV TEM (FEl, Netherlands) operated at 200 kV at desired magnification.

\section{Evaluation of encapsulation of curcumin, piperlongumine and quercetin in loaded NPs}

Supernatant (10 $\mu \mathrm{L}$, after separating out NPs) was evaluated for the encapsulation and loading of three molecules (Q, P and C) using HPLC (Waters, USA) instrument having auto sampler (Auto-2707) and UVVisible detector (PDA 2998) using analytical C-18 column (Waters, USA, $4.6 \times 250 \mathrm{~mm}$ ). HPLC method was validated using solvent acetonitrile (0.1\% TFA) and water (50:50) at $370 \mathrm{~nm}, 325 \mathrm{~nm}$ and $420 \mathrm{~nm}$ wavelength for $\mathrm{Q}, \mathrm{P}$ and $\mathrm{C}$ respectively. The wavelength is selected based on the existing method. The limits of detection (LOD) and limit of quantitation (LOQ) for Q, P and C was evaluated. The formula 2 and formula 3 were used to calculate the encapsulation efficiency and loading of molecules respectively.

$\mathrm{EE}(\%)=\frac{\text { (amount of curcumin,piperlongumin and quercetin entrapped) }}{\text { (total amount of curcumin,piperlongumin and quercetin in formulation) }} \times 100$

QPC loading $(\%)=\frac{\text { (mass of QPC entrapped in nanoparticles) }}{\text { (mass of nanoparticles recovered after lyophilisation) }} \times 100$

\section{In vitro release of quercetin, piperlongumine and curcumin in PBS buffer from loaded NPs}

From three formulation we have selected only freshly prepared and lyophilized 5 mg LEs-QPC-PLA NPs. LEs-QPC-PLA NPs were incubated in $10 \mathrm{~mL} 0.1 \mathrm{M}$ phosphate buffer/saline at physiological $\mathrm{pH}(\mathrm{pH} 7.4)$. To study the in vitro release kinetics, LEs-QPC-PLA NPs were continuously stirred by a magnetic stirrer at $37^{\circ} \mathrm{C}$. Q, P and $\mathrm{C}$ containing released sample were collected $(1 \mathrm{~mL})$ at $0,4,8,12$ and 24 h, lyophilized and again dissolved in acetonitrile $(1 \mathrm{~mL})$. The dissolved sample were centrifuged at $10000 \mathrm{rpm}$ for $20 \mathrm{~min}$ at $4^{\circ} \mathrm{C}$. The amount of $\mathrm{Q}, \mathrm{P}$ and $\mathrm{C}$ released (\%) at any time ' $\mathrm{t}$ ' was calculated using the formula 4 :

Cumulative release $(\%)=\frac{(\text { Released amount of } Q P C \text { at time } t)}{(\text { Total amount of } Q P C \text { at time } 0)} \times 100$

\section{Development and standardization of colon cancer model on animal male BALB/c mice}

Male BALB/c mice were procured from central animal house, Panjab University Chandigarh, India. All the experimental procedures were first approved from ethical committee and conducted according to the guidelines of Indian National Science Academy, New Delhi. The animals were treated with single intraperitoneal dose of DMH (20 mg/kg body weight). After one week, DSS (3\% w/v) was given in drinking water for one week followed by normal drinking water for two weeks. The animals were subjected to three such DSS cycles. The animal model was standardised for the development of colon cancer and observed through the histological section and staining under microscope (Nikon Eclipse 80i). 
Eight group of animal $(n=5)$ including control (DMH-DSS), pure molecules (QPC), LEs-PLA NPs (blank), LEs-S-PLA NPs (blank), LEs-QPC-PLA NPs (loaded) and LEs-S-QPC-PLA NPs (loaded) were used for the anticancer study. The dose of curcumin was selected as standard $(40 \mathrm{mg} / \mathrm{kg})$ dose from the already published paper. And we have reduced the dose half to set $20 \mathrm{mg} / \mathrm{kg}$ due to three molecules available in the loaded formulation. Dose were (Pure molecules, blank and loaded NPs) given in saline (200 $\mu \mathrm{L}$ for each animals) via oral route for 15 days at alternate days. Only saline was given to the controller group (DMH-DSS) at alternate date for the same period (15 days).

\section{Detection of quercetin, piperlongumine and curcumin in different organs using HPLC}

After the completion of dose, animals were sacrifice and liver, kidney, colon, serum were analysed for the presence of quercetin, piperlongumine and curcumin by HPLC. Each organ $(20 \mathrm{mg})$ were crushed in acetonitrile $(1 \mathrm{~mL})$ with mortar and pestle, filtered and $10 \mu \mathrm{L}$ of each was injected into the HPLC with help of auto sampler. The presence of the $\mathrm{Q}, \mathrm{P}$ and $\mathrm{C}$ was recorded and analysed.

\section{Anticancer activity of pure molecules, blank and loaded NPs using histopathology in colon, liver, kidney and serum}

The animals were sacrificed by cervical dislocation after completion of dose and then their colon, liver, kidney and serum were collected for further study. Buffer washed organs were preserved in the buffered formalin (10\%) and fixed into the wax blocks for sectioning (4-5 $\mu \mathrm{m})$. The fixed sections were stained with standard haematoxylin and eosin (H\&E) staining using standard protocol. The well stained oven dried section slides were observed under microscope (Nikon 80i) and evaluated for the presence of adenoma, hyperplasia and cancer in situ.

\section{Results And Discussion}

\section{Synthesis of blank and loaded NPs}

Banyan ( $F$. benghalensis) grows in hot tropical climates and its aerial parts and roots are used as ingredients in medicine [11]. The aqueous leaf extract of various plants were used to enhance the medicinal value of NPs [12]. In fact, such natural systems are among the most promising for the developments in nanomedicine, which has grown exponentially: from simple nanoparticles loaded with drugs to multifunctional nanoparticles and could be possible to target to specific cancer cells through binding to unique cell-surface proteins [13]. Still drug delivery via NPs and conventional clinical drug delivery have highly focused around synthetic molecules and use of conventional method for the synthesis of NPs.

The NPs were synthesized from the reported methods with slight modifications dealing with the toxicity and drug delivery [14]. The plant mediated NPs were synthesized to minimise the toxicity issues associated with chemicals used in their synthesis and efforts have been done to enhance the uptake and long term survival of NPs in the systemic circulation. The leaf extract of Banyan is a rich source of many 
therapeutic molecules and secondary metabolites [15]. These molecules may get loaded during the synthesis of NPs using solvent evaporation method [16]. Polymer PLA and natural molecules can be easily mixed with each other in DCM. The sonication homogenise the mixture and addition of $4 \mathrm{~mL}$ of LE and sonication change the reaction mixture into big droplet of emulsion [17] while dilution of reaction mixture with distilled water well-distributed the droplet containing NPs. The evaporation of DCM by rotavapour matured the NPs under the vacuum. The addition of biogenic molecules stabilized the NPs and did not interfere with the encapsulation [12]. The blank and loaded NPs were easily redistributed in the distilled water after centrifugation.

\section{Physical characterisation of LEs-S-PLA NPs, LEs-PLA NPs, LEs-QPC-PLA NPs and LEs-S-QPC-PLA NPs}

HRTEM is a powerful instrument to characterise the morphology and size of NPs [18]. PLA polymeric NPs are generally round in shape when formed by solvent evaporation method [19]. The synthesized blank NPs were found round in shape and their size was inhomogeneous. The blank and loaded NPs were observed at higher resolution (10 nm) to know the crystal nature of NPs (Fig. 1). The blank and loaded NPs were assembled into crystal structure at the time of synthesis [20] and were observed at the higher resolution in HRTEM. The size, shape and coating on the surface of the NPs play important role in systemic circulation and half-life during in vivo study [17]. The size of the synthesized NPs were in application range and suitable for the drug delivery.

\section{Evaluation of quercetin, piperlongumine and curcumin encapsulation using HPLC}

The amount of molecules loaded in the NPs is important for the drug delivery [21]. High drug loaded polymeric NPs are recently explored to target cancer cells and loading of more than one molecules in NPs is not explored on the cancer model. The encapsulation efficiency of Q, P and C in LEs mediated PLA NPs was measured using modified HPLC method $[22,23]$. The supernatant of LEs-QPC-PLA NPs obtained by centrifugation was filtered via $0.22 \mu \mathrm{m}$ filter. The supernatant $(1 \mathrm{~mL})$ was lyophilized and re-dissolved in acetonitrile and injected in HPLC (Waters, coupled with diode array detector 2998) using auto sampler and reverse phase C-18 column and a mobile phase containing acetonitrile and water (50:50, v/v) was used with a flow rate of $1 \mathrm{~mL} / \mathrm{min}$ and detection wavelength was 370,325 and $420 \mathrm{~nm}$ (Fig. 2). The limits of detection (LOD) and limits of quantitation (LOQ) for Q by HPLC method were $0.0004842 \mathrm{mg} / \mathrm{mL}$ and $0.0004842 \mathrm{mg} / \mathrm{mL}$ respectively. While for $P$ the HPLC method showed LOD and LOQ of 0.000001525 $\mathrm{mg} / \mathrm{mL}$ and $0.0003062 \mathrm{mg} / \mathrm{mL}$, respectively while for curcumin LOD was $0.00006103 \mathrm{mg} / \mathrm{mL}$ and LOQ was $0.00001525 \mathrm{mg} / \mathrm{mL}$. The calibration curve was plotted using different concentrations of $\mathrm{Q}$ (0.01562-0.25 mg), P (0.01562-0.25 mg) and C (0.03125-0.5 mg). The calibration curve was found linear and correlation coefficient was calculated as 0.9979 for $Q$ and 0.9998 for $P$ and 0.9995 for $C$. The average encapsulation efficiency of $Q, P$ and $C$ was $93.8 \%, 94.9 \%$ and $85.3 \%$ respectively. The average loading of Q, P and C was $16 \%, 14 \%$ and $15 \%$ respectively and total loading of three molecules was nearly $50 \%$ showing good loading of the three molecules.

\section{In vitro release of curcumin, piperlongumine and quercetin in PBS buffer from LEs-QPC-PLA NPs}


The physiological pH provides simulating conditions for the release of molecules from the loaded NPs [24]. The polymeric NPs have been used in the drug delivery for their slow and sustained release. The incubation of loaded NPs with PBS buffer allows the release of three molecules (Q, P and C) into the buffer at physiological $\mathrm{pH}$. The release of curcumin was found to be burst and $32 \%$ at $0 \mathrm{~h}$ while \%age (percentage) release of the quercetin and piperlongumine were $18.5 \%$ and $28 \%$ respectively (Fig. 3 ). After $0 \mathrm{~h}$, the release of the three molecules was slow and sustained. The maximum \%age release of the quercetin, piperlongumine and curcumin was $42 \%, 36.5 \%$ and $60.5 \%$ respectively after $24 \mathrm{~h}$. The molecules $\mathrm{Q}, \mathrm{P}$ and $\mathrm{C}$ were released independently and did not show any kind of release interactions with each other. The burst release of curcumin might be due to more surface loading that was observed with naked eye. The release of molecules from PLA NPs during in vivo conditions is different which could be influenced by attachment of vital molecules on the surface of NPs [19].

\section{Distribution of quercetin, piperlongumine and curcumin in different organs and their detection by HPLC}

Colon cancer is a growing problem in the society and there are limited options of chemotherapy available in the medicine field [25]. Few approaches are used to treat the cancer using synthetic molecules and nano-formulations but the issues of toxicity and drug delivery at the cancer site are still challenging [26]. The oral route for the delivery of NPs loaded with natural molecules is reported to be safe and easier [27]. The NPs have the potential to cross the mucous layer and deliver the molecules inside the body [28]. The systemic circulation of released molecules from NPs depends upon nature of the molecules and their binding to other vital moieties or proteins [29]. Enhancing the uptake and distribution of NPs in systemic circulation depends upon various factors like charge, size and surface morphological properties. The loading of Q, P and C was good and the surface of blank and loaded NPs coated with LEs and dextrose enhanced the bioavailability and reduced toxicity in the colon cancer. The pure molecules $(\mathrm{Q}, \mathrm{P}$ and $\mathrm{C})$ were detected in the liver but not detected in colon and kidney, however, $Q$ and $P$ were detected in serum and $\mathrm{C}$ was not found. The LEs formulations loaded with NPs i.e. LEs-QPC-PLA NPs showed promising results to maintain the natural molecules in different organs after $8 \mathrm{~h}$. LEs-QPC-PLA NPs maintained the detectable concentration of Q, P and C in liver, kidney, serum and colon after $8 \mathrm{~h}$ of administration. $\mathrm{P}$ and $\mathrm{C}$ were not detected in kidney and serum. In case of LEs-S-QPC-PLA NPs formulations, Q, P and C were detected in kidney, liver and colon (Table 1 ) while $C$ was not accessible in serum. The availability of Q, P and $\mathrm{C}$ in different organs depends upon various factors like their oral absorption, release from the NPs, solubility, amount of dose, time period of detection and biocompatibility of molecules with vital molecules and proteins [30,31]. It can be concluded that all three molecules were organic soluble therefore the NPs distributed these molecules in the vital organs. 
Table 1

Three Formulations, Pure Molecules (Quercetin, Piperlongumine And Curcumin), LEs-QPC-PLA NPs and LEs-S-QPC-PLA NPs) and Organ Wise (Kidney, Liver, Serum And Colon) Detection Of Molecules After 8 Hour Delivery Using HPLC

\begin{tabular}{|c|c|c|c|c|}
\hline \multirow[t]{2}{*}{$\begin{array}{l}\text { Oral dosing }(20 \mathrm{mg} / \mathrm{kg}) \text { of } \\
\text { curcumin }\end{array}$} & \multirow[t]{2}{*}{ Molecules } & $\begin{array}{l}\text { Pure } \\
\text { Molecules }\end{array}$ & \multirow[t]{2}{*}{$\begin{array}{l}\text { LEs-QPC-PLA } \\
\text { NPs }\end{array}$} & \multirow[t]{2}{*}{$\begin{array}{l}\text { LES-S-QPC-PLA } \\
\text { NPS }\end{array}$} \\
\hline & & $(Q, P, C)$ & & \\
\hline \multirow[t]{3}{*}{ Kidney } & Q & ND & $\mathrm{D}$ & D \\
\hline & $P$ & ND & ND & D \\
\hline & C & ND & $D$ & D \\
\hline \multirow[t]{3}{*}{ Liver } & $\mathrm{Q}$ & D & $D$ & D \\
\hline & $P$ & D & $D$ & D \\
\hline & $C$ & D & $\mathrm{D}$ & D \\
\hline \multirow[t]{3}{*}{ Serum } & $\mathrm{Q}$ & D & $D$ & D \\
\hline & $P$ & D & $\mathrm{D}$ & D \\
\hline & C & ND & ND & ND \\
\hline \multirow[t]{3}{*}{ Colon } & Q & ND & $\mathrm{D}$ & D \\
\hline & $P$ & ND & $\mathrm{D}$ & D \\
\hline & C & ND & $D$ & D \\
\hline
\end{tabular}

\section{Anticancer activity of Q, P, C and loaded NPs using histopathology}

Colon cancer is presently the emphasized problem due to unhealthy food habits and toxicity in the environment [32]. Numerous approaches have been used to treat the colon cancer but there is a lot of lacking towards NPs led drug delivery in a combinatorial way [33]. Q, P and C are well known anticancer, anti-inflammatory, antioxidant etc. [3, 4]. However, the problem of their absorption and reaching up to the target during in vivo study is quite challenging. The three natural molecules were found to reduce the toxicity issues in animal studies. The researchers have synthesized the individual NPs of Q, P and C [3436]. The combinatorial approach of action of more than one molecules on cancer was quite good in few studies [37]. However in present work, we have synthesized three molecules in a single biodegradable NPs and their performance was improved in a combinatorial way that might be due to the leaf nanocoating. The extract of Banyan leaf is highly efficient and have already been used in traditional therapies [11]. The histopathology of colon cancer showed features of polyploidy, adenoma, hyperplasia and cancer in situ in the control animals of DMH-DSS. The blank NPs could not show prominent results to 
control the cancer while all the loaded NPs reduced the tumor and improved the physiology of colon cancer tissues that was observed under the microscope (Figs. 4, 5, 6). The loaded LEs-QPC-PLA NPs showed good anticancer properties as compared to the blank NPs and pure molecules. The growth of cancer cells require more nutrient for cell metabolism and growth. The coating of sugar on the surface of NPs might result in their easy uptake by cancer cells [38]. The sugar coated LEs-S-QPC-PLA NPs exhibited prominent results and reduced adenoma and improved overall architecture of crypt as compared to the LEs-QPC-PLA NPs. The sugar coating on NPs make them easily cross the membrane and it could enhanced the bioavailability of $\mathrm{Q}, \mathrm{P}$ and $\mathrm{C}$ in the tumor area. The group treated with sugar coated LEs-SQPC-PLA NPs distinctly recovered the normal crypt architecture and restored the goblet cell population (Figs. 4, 5, 6) in comparison to the LEs-QPC PLA NPs and pure molecules (Q, P, C). The survival of the NPs in the systemic circulation depends upon the compatibility of NPs with vital molecules [39]. The NPs maintained the presence of $\mathrm{Q}, \mathrm{P}$ and $\mathrm{C}$ even after $8 \mathrm{~h}$ in different organs showing reduced tumors and thus enhanced anticancer activity (Figs. 4, 5,6). The pure molecules also showed promising results but were not much effective as compared to the loaded NPs as reported by various researchers. Therefore present study showed the improvement in the performance of $\mathrm{Q}, \mathrm{P}$ and $\mathrm{C}$ molecules using NPs, minimize their toxicity using coating on the surface of NPs, high drug high loading and combinatorial natural molecules approach.

The liver and kidney got affected by the DMH-DSS shown by histopathology study. The liver showed inflammation in the control and blank NPs (DMH-DSS, LEs-PLA NPs, LEs-S-PLA NPs). However some recovery was observed by treatment with loaded NPs LEs-QPC-PLA NPs and LEs-S-QPC-PLA NPs but inflammation was still observed in sections. The histopathology of kidney was studied and nephrotoxicity was observed in control, blank NPs and Q, P and C molecules represented by green and red circle (damage) a damaged in Bowman capsule and inflammation (Figs. 4, 5, 6). The loaded NPs were quite recovered from the nephrotoxicity. To analyse the renal and hepatotoxicity more study is required to confirm the data. The three molecules have anticancer potential and are also effective to some extent against hepatic and nephrotoxicity induced by the DMH-DSS in BALB/c mice. The present study is a lead for making effective formulations based on NPs using natural molecules in a combinatorial way.

\section{Conclusion}

The NPs are designed to enhance the performance of loaded natural molecules. The present study was designed for generating nano formulations having combination of natural molecules. The study was carried out to reduce the toxicity and to enhance the effectiveness of treatment in many folds by oral route using the combinatorial approach of natural molecules having LEs as a surfactant and stabilizer in NPs synthesis. One of the fundamental motivations behind this examination is to improve the bioavailability of quercetin, piperlongumine and curcumin for colon cancer treatment. The results from LEs mediated NPs were promising in terms of surface coating, high loading of molecules, and in vitro release in sustainable way and for their anticancer potential in a therapeutic way. In the formulations the sugar coated NPs enhanced the bio-distribution of natural molecules in colon cancer and exhibited prominent anticancer prospective. The histopathology study showed the recovery in liver and kidney 
organs. Therefore, it can be concluded that the present nano-formulation can be a good lead against the colon cancer. However, there is a need for more standardization like targeting and coating with different agents to maximise the uptake of NPs.

\section{Declarations}

\section{Compliance with ethical standards}

All experimental protocols were first approved by Institutional Ethics Committee (PU/IAEC/S/14/47) of Panjab University, Chandigarh, India and conducted according to the guidelines of Indian National Science Academy for the use and care of experimental animals.

\section{Availability of data and material}

Additional figures are included in the supplementary Material

\section{Funding information}

This work was supported financially by Department of Science \& Technology (Science and Engineering Research Board, PDF/2016/ 002438) to author RDY.

\section{Authors' contributions}

All authors contributed to the preparation of manuscript. All authors read and approved the final manuscript.

\section{Conflict of interest}

The authors declare that they have no conflict of interest.

\section{Acknowledgements}

The author RDY is thankful to the Department of Science \& Technology (Science and Engineering Research Board) for providing financial support.

\section{Consent for publication}

Not applicable.

\section{References}

1. Yue Y, Ye K, Lu, J, Wang X, Zhang S, Liu L, Yang B, Nassar K, Xu X, Pang X, Lv J. Probiotic strain Lactobacillus plantarum $Y Y C-3$ prevents colon cancer in mice by regulating the tumour microenvironment. Biomed Pharmacother. 2020;127:110159. 
2. Satapathy SR, Sjölander A. Cysteinyl leukotriene receptor 1 promotes 5 -fluorouracil resistance and resistance-derived stemness in colon cancer cells. Cancer Lett. 2020;488:50-62.

3. Zhao J, Fang Z, Zha Z, Sun Q, Wang H, Sun M, Qiao B. Quercetin inhibits cell viability, migration and invasion by regulating miR-16/HOXA10 axis in oral cancer. Eur J Pharmacol. 2019;847:11-8.

4. Moradi-Marjaneh R, Hassanian, SM, Rahmani F, Aghaee-Bakhtiari SH, Avan A, Khazaei M. Phytosomal curcumin elicits anti-tumor properties through suppression of angiogenesis, cell proliferation and induction of oxidative stress in colorectal cancer. Curr Pharm Des. 2018;24:4626-38.

5. Patel SS, Acharya A, Ray RS, Agrawal R, Raghuwanshi R, Jain P. Cellular and molecular mechanisms of curcumin in prevention and treatment of disease. Crit Rev Food Sci Nutr. 2020;60:887-939.

6. Lodi A, Saha A, Lu X, Wang B, Sentandreu E, Collins M, Kolonin MG, DiGiovanni J, Tiziani S. Combinatorial treatment with natural compounds in prostate cancer inhibits prostate tumor growth and leads to key modulations of cancer cell metabolism. NPJ Precis Oncol. 2017;1:18.

7. Ratovitski E. Anticancer natural compounds: Molecular mechanisms and functions. Part I. Curr Genomics. 2017;18:105.

8. Rayan A, Raiyn J, Falah M. Nature is the best source of anticancer drugs: Indexing natural products for their anticancer bioactivity. PLoS One. 2017;12:e0187925.

9. Zhou P, Qin J, Zhou C, Wan G, Liu Y, Zhang M, Yang X, Zhang N, Wang Y. Multifunctional nanoparticles based on a polymeric copper chelator for combination treatment of metastatic breast cancer. Biomaterials. 2019;195:86-99.

10. Singh P, Kim YJ, Zhang D, Yang DC. Biological synthesis of nanoparticles from plants and microorganisms. Trends Biotechnol. 2016;34:588-99.

11. Panday DR., Rauniar GP. Effect of root-extracts of Ficus benghalensis (Banyan) in memory, anxiety, muscle co-ordination and seizure in animal models. BMC Complement Altern Med. 2016;16:429.

12. Yadav R, Kumar D, Kumari A, Yadav SK. PLA nanovectors with encapsulated betulin: plant leaf extract-synthesized nanovectors are more efficacious than PVA-synthesized nanovectors. Biotechnol Lett. 2016;38:259-69.

13. Zhang M, Ma Y, Wang Z, Han Z, Gao W, Zhou Q, Gu Y. A CD44-targeting programmable drug delivery system for enhancing and sensitizing chemotherapy to drug-resistant cancer. ACS Appl Mater Interfaces. 2019;11:5851-61.

14. Kumari A, Kumar V, Yadav SK. Plant extract synthesized PLA nanoparticles for controlled and sustained release of quercetin: A green approach. PLoS One. 2012;7:e41230.

15. El-hawary SS, Ali ZY, Younis IY. Hepatoprotective potential of standardized Ficus species in intrahepatic cholestasis rat model: Involvement of nuclear factor- $\mathrm{KB}$, and Farnesoid $\mathrm{X}$ receptor signaling pathways. J Ethnopharmacol. 2019;231:262-74.

16. Ren Y, Li X, Han B, Zhao N, Mu M, Wang C, Du Y, Wang Y, Tong A, Liu Y, Zhou L, You C, Guo G. Improved anti-colorectal carcinomatosis effect of tannic acid co-loaded with oxaliplatin in nanoparticles encapsulated in thermosensitive hydrogel. Eur J Pharm Sci. 2019;128:279-89. 
17. Yadav R, Kumar D, Kumari A, Yadav SK. Encapsulation of catechin and epicatechin on BSA NPs improved their stability and antioxidant potential. EXCLI J. 2014;13:331-346.

18. Jamil A, Mirza MA, Anwer MK, Thakur PS, Alshahrani SM, Alshetaili AS, Telegaonkar S, Panda AK, Iqbal Z. Co-delivery of gemcitabine and simvastatin through PLGA polymeric nanoparticles for the treatment of pancreatic cancer: in-vitro characterization, cellular uptake, and pharmacokinetic studies. Drug Dev Ind Pharm. 2019;45:745-53.

19. Sun Z, Cheng K, Yao Y, Wu F, Fung J, Chen H, Ma X, Tu Y, Xing L, Xia L, Cheng Z. Controlled nano-bio interface of functional nanoprobes for in vivo monitoring enzyme activity in tumors. ACS Nano. 2019;13:1153-1167.

20. Moreau LM, Ha DH, Zhang H, Hovden R, Muller DA, Robinson RD. Defining crystalline/amorphous phases of nanoparticles through X-ray absorption spectroscopy and X-ray diffraction: The case of nickel phosphide. Chem Mater. 2013;25:2394-2403.

21. Sajjad M, Khan MI, Naveed S, Ijaz S, Qureshi OS, Raza SA, Shahnaz G, Sohail MF. Folatefunctionalized thiomeric nanoparticles for enhanced docetaxel cytotoxicity and improved oral bioavailability. AAPS PharmSciTech. 2019;20:81.

22. Kamal YT, Singh M, Salam S, Ahmad S. Simultaneous quantification of piperlongumine and piperine in traditional polyherbal formulation using validated HPLC method. Acta Chromatogr. 2016;28:489500.

23. Ang LF, Yam MF, Fung YTT, Kiang PK, Darwin Y. HPLC method for simultaneous quantitative detection of quercetin and curcuminoids in traditional chinese medicines. $J$ Pharmacopuncture. 2014;17:36-49.

24. Yadav R, Kumar D, Kumari A, Yadav SK. Encapsulation of podophyllotoxin and etoposide in biodegradable poly-D,L-lactide nanoparticles improved their anticancer activity. J Microencapsul. 2014;31:211-19.

25. Ochiai T, Nishimura K, Watanabe T, Kitajima M, Nakatani A, Nagayasu K, Sakuyama N, Sato T, Kishine K, Abe Y, Nagaoka I. Impact of primary tumor location as a predictive factor in patients suffering from colorectal cancer treated with cytotoxic anticancer agents based on the collagen gel droplet-embedded drug sensitivity test. Oncol Lett. 2019;17:1842-50.

26. Sousa AR, Oliveira MJ, Sarmento B. Impact of CEA-targeting nanoparticles for drug delivery in colorectal cancer. J Pharmacol Exp Ther. 2019;370:657-70.

27. Diab R, Jaafar-Maalej C, Fessi H, Maincent P. Engineered nanoparticulate drug delivery systems: the next frontier for oral administration. AAPS J. 2012;14:688-702.

28. Date AA, Hanes J, Ensign LM. Nanoparticles for oral delivery: Design, evaluation and state-of-the-art. J Control Release. 2016;240:504-26.

29. Din FU, Aman W, Ullah I, Qureshi OS, Mustapha O, Shafique S, Zeb A. Effective use of nanocarriers as drug delivery systems for the treatment of selected tumors. Int J Nanomedicine. 2017;12:7291-309.

30. Ghalandarlaki N, Alizadeh AM, Ashkani-Esfahani S. Nanotechnology-applied curcumin for different diseases therapy. BioMed Res Int. 2014;2014:394264. 
31. Farazuddin M, Dua B, Zia Q, Khan AA, Joshi B, Owais M. Chemotherapeutic potential of curcuminbearing microcells against hepatocellular carcinoma in model animals. Int $\mathrm{J}$ Nanomedicine. 2014;9:1139-52.

32. Arnold M, Sierra MS, Laversanne M, Soerjomataram I, Jemal A, Bray F. Global patterns and trends in colorectal cancer incidence and mortality. Gut. 2017;66:683-691.

33. Serrano D, Bonanni B, Brown K. Therapeutic cancer prevention: achievements and ongoing challenges - a focus on breast and colorectal cancer. Mol Oncol. 2019;13:579-90.

34. Han Q, Wang X, Cai S, Liu X, Zhang Y, Yang L, Wang C, Yang R. Quercetin nanoparticles with enhanced bioavailability as multifunctional agents toward amyloid induced neurotoxicity. J Mater Chem B. 2018;6:1387-93.

35. Wang W, Chen T, Xu H, Ren B, Cheng X, Qi R, Liu H, Wang Y, Yan L, Chen S, Yang Q, Chen C. Curcuminloaded solid lipid nanoparticles enhanced anticancer efficiency in breast cancer. Molecules. 2018;23:1578.

36. Liu Q, Zhao D, Zhu X, Chen H, Yang Y, Xu J, Zhang Q, Fan A, Li N, Guo C, Kong Y, Lu Y, Chen X. Coloaded nanoparticles of paclitaxel and piperlongumine for enhancing synergistic antitumor activities and reducing toxicity. J Pharm Sci. 2017;106:3066-75.

37. Mishra J, Drummond J, Quazi SH, Karanki SS, Shaw JJ, Chen B, Kumar N. Prospective of colon cancer treatments and scope for combinatorial approach to enhanced cancer cell apoptosis. Crit Rev Oncol Hematol. 2013;86:232-50.

38. Das MR, Bag AK, Saha S, Ghosh A, Dey SK, Das P, Mandal C, Ray S, Chakrabarti S, Ray M, Jana SS. Molecular association of glucose-6-phosphate isomerase and pyruvate kinase M2 with glyceraldehyde-3-phosphate dehydrogenase in cancer cells. BMC Cancer. 2016;16:152.

39. Samani E, Seraj S, Naderimanesh Z, Khajeh HK, Rastaghi ARE, Droudi T, Kolivand P, Kazemi H, Asghari SM. Controlled release of an endostatin peptide using chitosan nanoparticles. Chem Biol Drug Des. 2017;90:417-24.

\section{Figures}



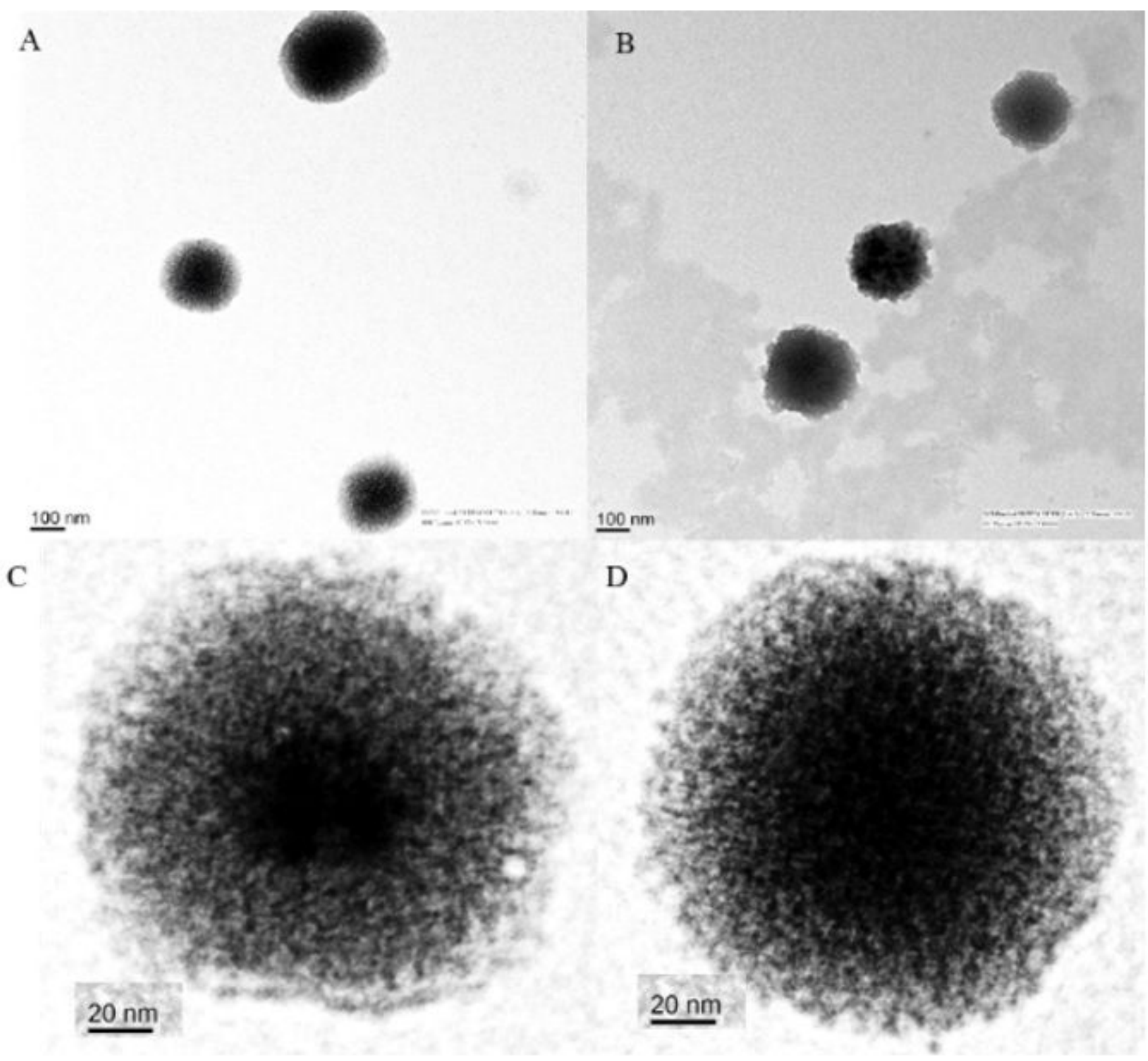

D

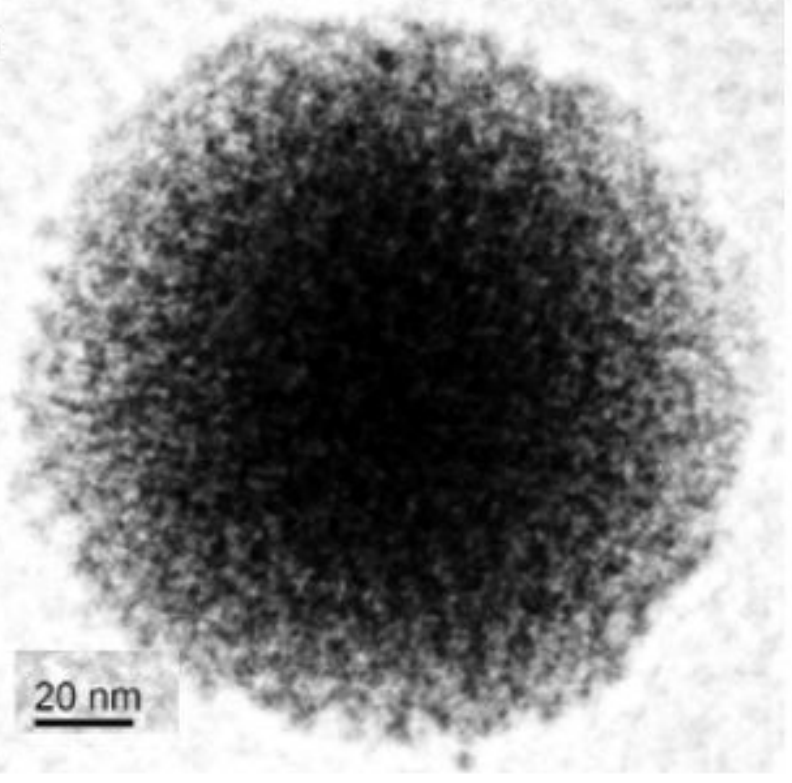

Figure 1

Characterization of LEs PLA NPs and LEs-QPC PLA NPs by HRTEM. Average HRTEM size of the LEs PLA NPs and LEs-QPC PLA NPs was $270 \mathrm{~nm}$ and $190 \mathrm{~nm}$ respectively 

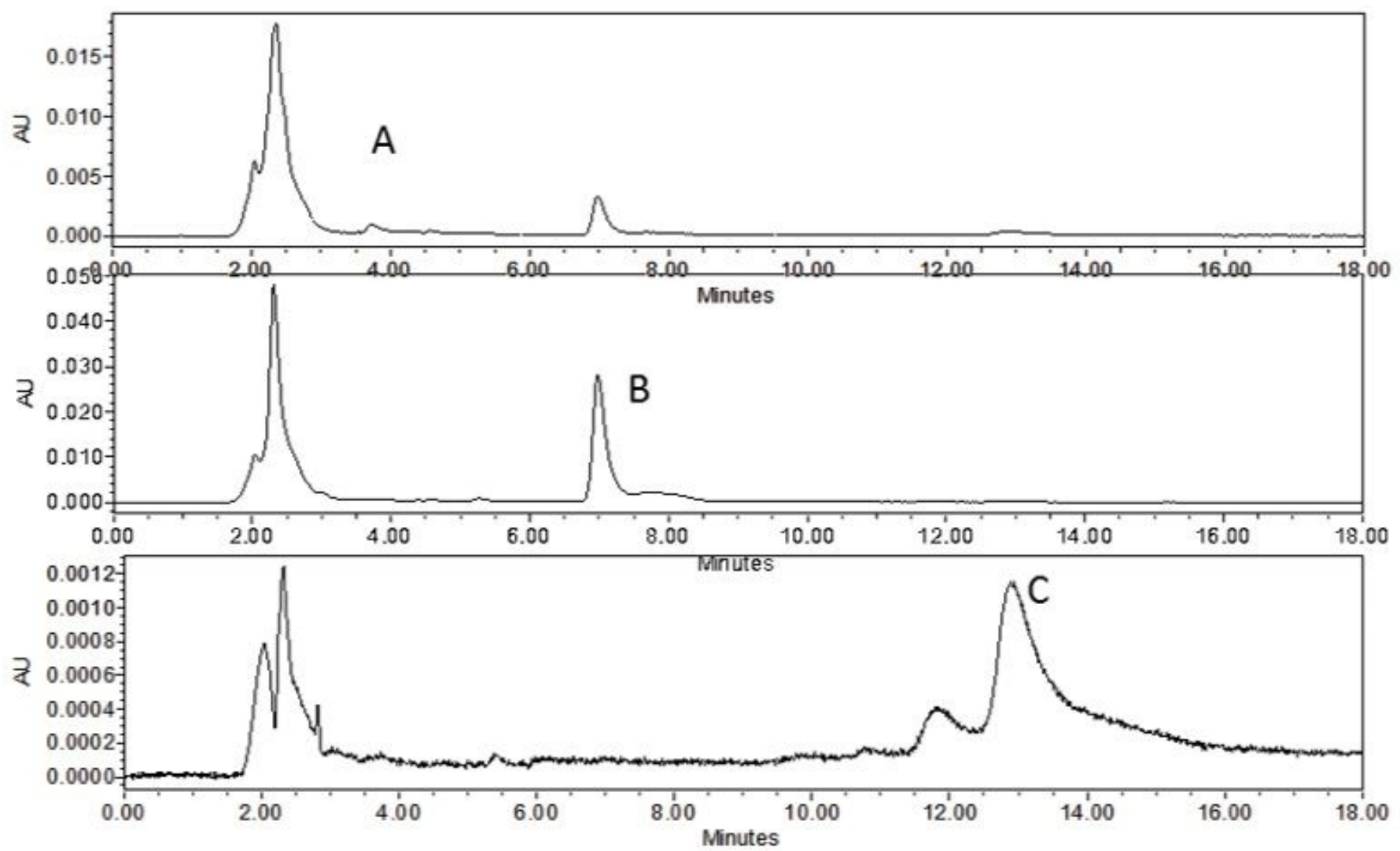

Figure 2

Evaluation of average encapsulation of quercetin, piperlongumine and curcumin in PLA NPs by HPLC. HPLC chromatograms of (A) quercetin, (B) piperlongumine and (C) curcumin 


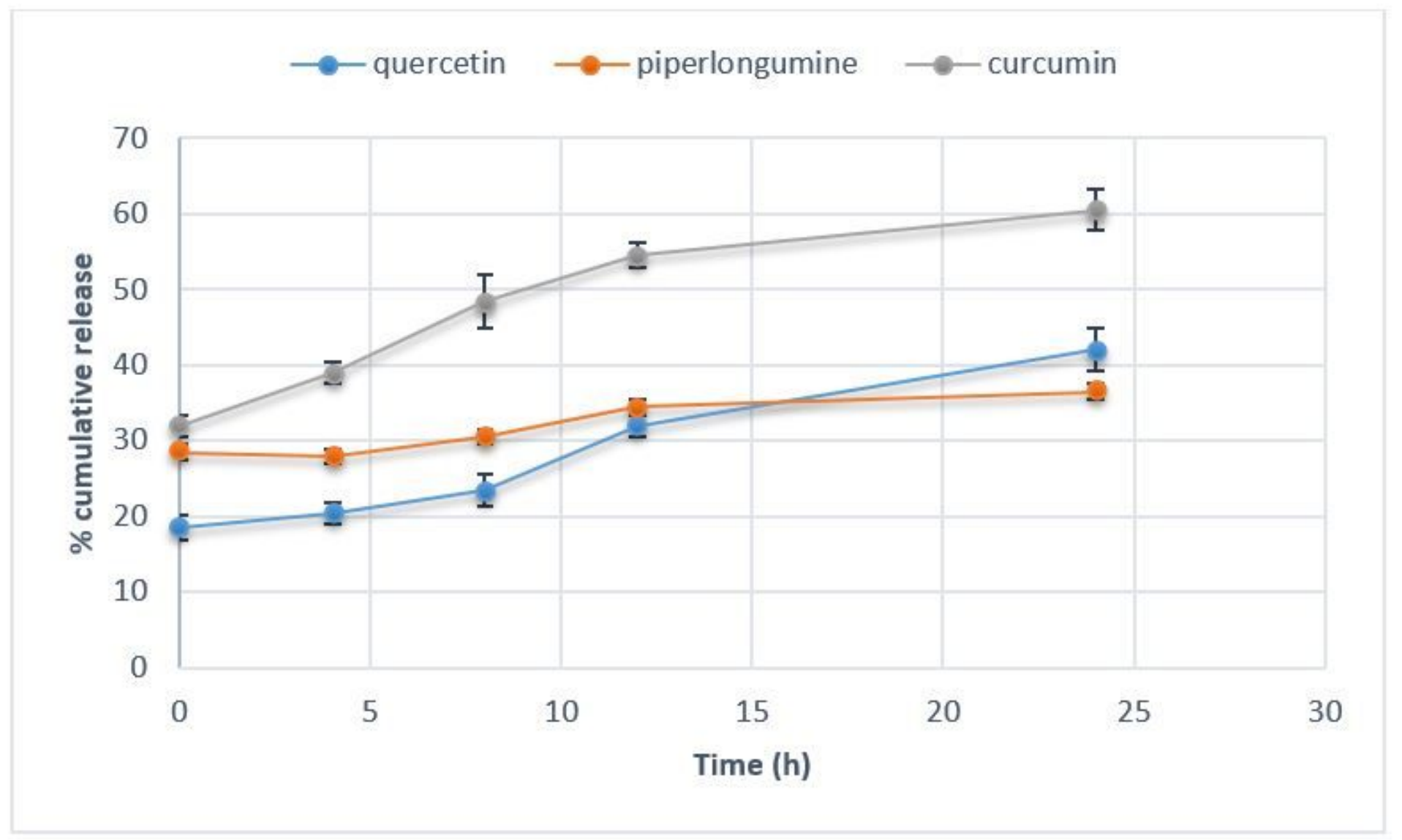

\section{Figure 3}

Average In vitro release of quercetin, piperlongumine and curcumin found maximum after $24 \mathrm{~h}$ as $42 \%$, $36.5 \%$ and $60.5 \%$ respectively
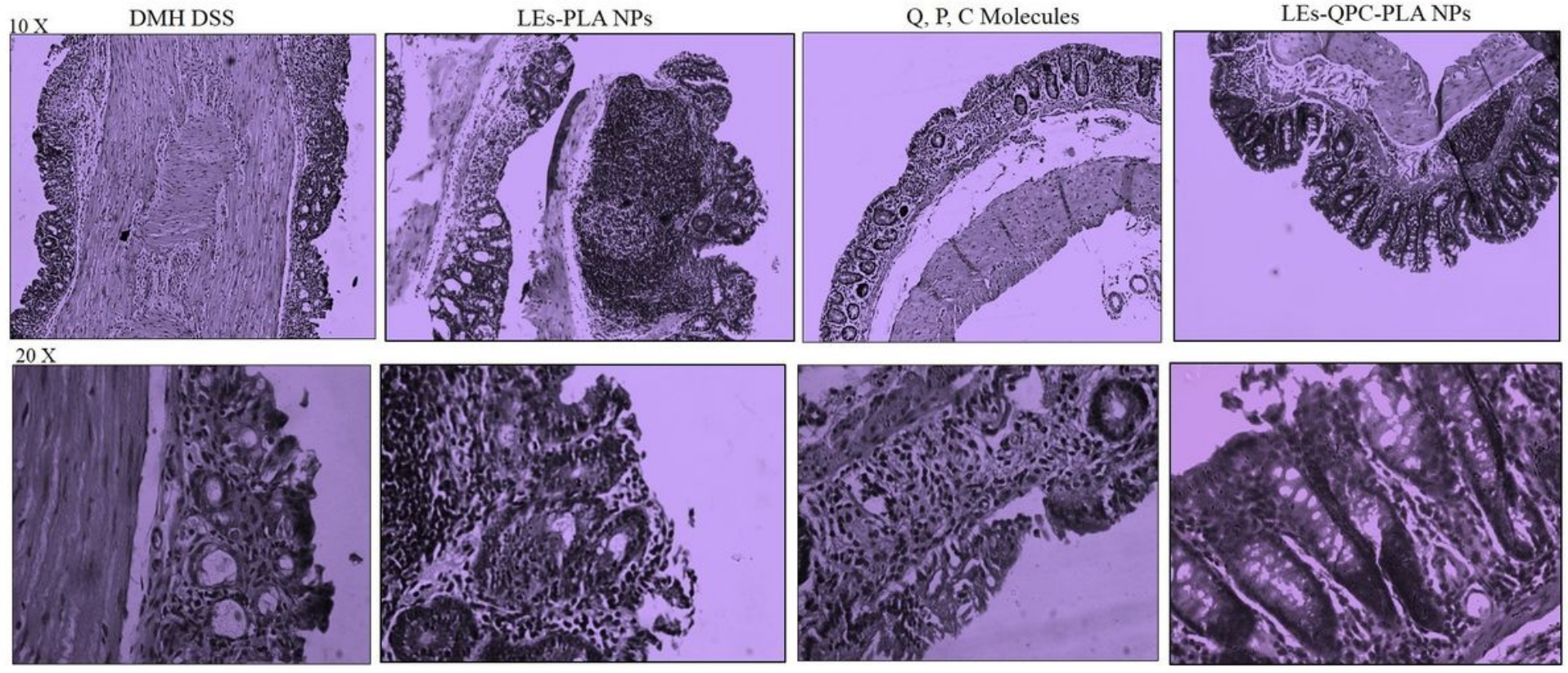

Figure 4 
Histopathology of colon tissue after dosing for 15 days @ alternate days; formulations: DMH-DSS (Control), LEs-PLA NPs, Q, P, C molecules and LEs-QPC-PLA NPs
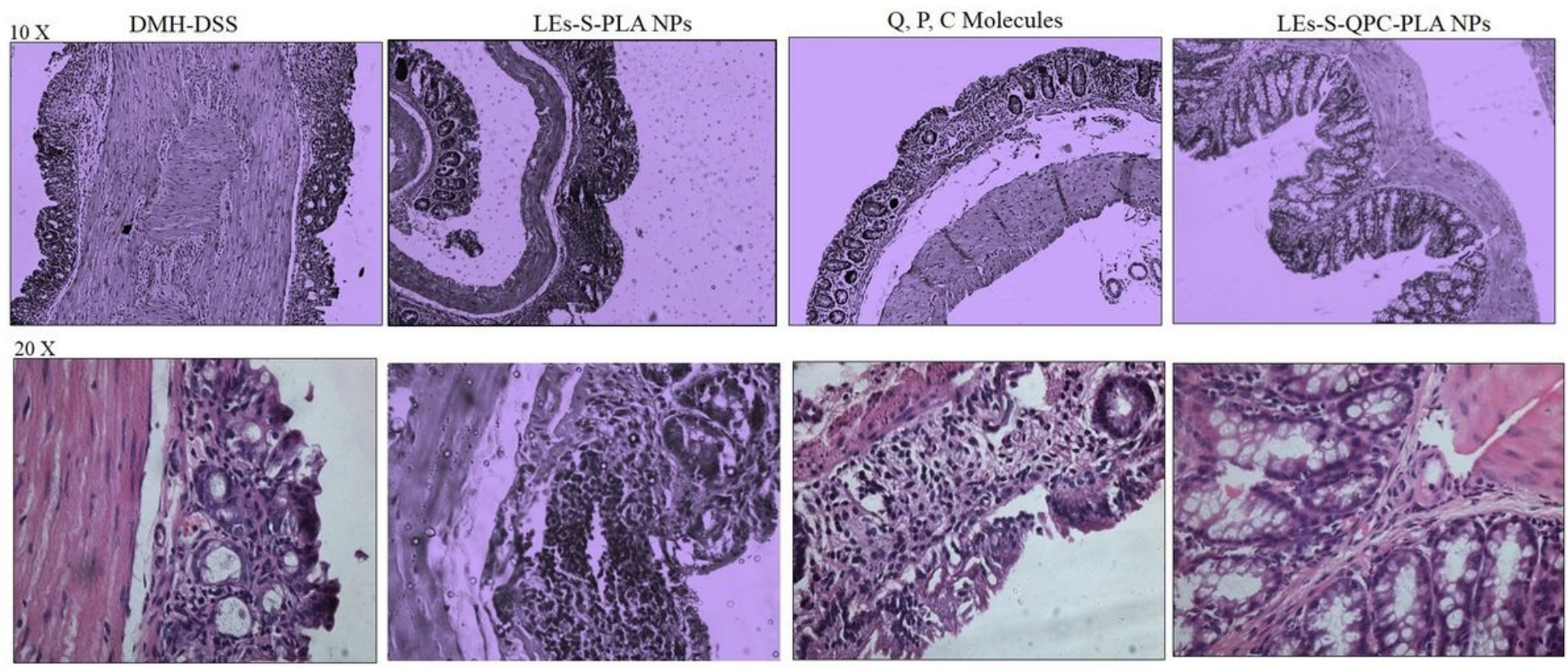

\section{Figure 5}

Histopathology of colon tissue after dosing for 15 days @ alternate days; formulations: DMH-DSS (Control), LEs-S-PLA NPs, only Q, P, C molecules and LEs-S-QPC-PLA NPs

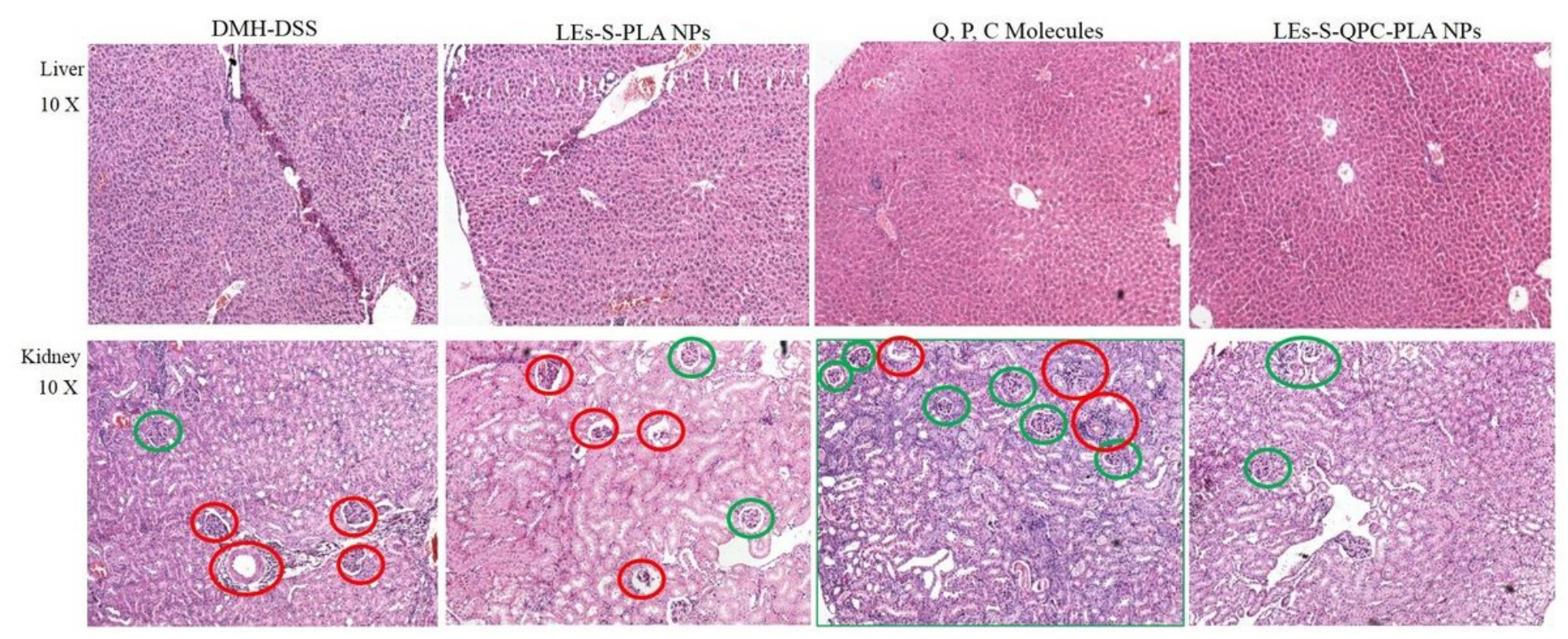

\section{Figure 6}

Histopathology of liver and kidney tissues after dosing for 15 days @ alternate days in colon cancer model (BALB/c mice); formulations: DMH-DSS (Control), LEs-S-PLA NPs, only Q, P, C molecules and LEsS-QPC-PLA NPS 


\section{Supplementary Files}

This is a list of supplementary files associated with this preprint. Click to download.

- GraphicalAbstract.docx

- SupplementaryFig.docx 